\title{
Fibonacci-Like Polynomials and Some Identities
}

\author{
Bijendra Singh $^{1}$, Shikha Bhatnagar ${ }^{1}$, Omprakash Sikhwal $^{2}$ \\ School of Studies in Mathematics, Vikram University Ujjain, India ${ }^{1}$ \\ Department of Mathematics, Mandsaur Institute of Technology, Mandsaur, India ${ }^{2}$ \\ *Corresponding author E-mail: suhani_bhatnagar@rediffmail.com
}

\begin{abstract}
The Fibonacci sequence and Fibonacci polynomials are famous for possessing wonderful and amazing properties and identities. In this paper, Fibonacci-Like polynomials (FLP) is introduce and define by $s_{n}(x)=x s_{n-1}(x)+s_{n-2}(x), n \geq 2$ with $s_{0}(x)=2$ and $s_{1}(x)=2 x$. Also some basic identities are presented and derived by standard method.
\end{abstract}

Keywords: Fibonacci polynomials, Fibonacci-Like polynomials, Binet's formula.

\section{Introduction}

It is well-known that the Fibonacci polynomials and Lucas polynomials are closely related and widely investigated. Fibonacci polynomials appear in different frameworks. These polynomials are of great importance in the study of many subjects such as algebra, geometry, combinatorics, approximation theory, statistics and number theory itself. Moreover these polynomials have been applied in every branch of mathematics. Fibonacci polynomials are special cases of Chebyshev polynomials and have been studied on a more advanced level by many mathematicians. The Fibonacci polynomials appear as the elements of $Q$ - matrix.

The Fibonacci polynomials [4] are defined by

$f_{n}(x)=x f_{n-1}(x)+f_{n-2}(x), n \geq 2$ with $f_{0}(x)=0, f_{1}(x)=1$.

Similarly, The Lucas polynomials [4] are defined by

$l_{n}(x)=x l_{n-1}(x)+l_{n-2}(x), n \geq 2$ with $l_{0}(x)=2, l_{1}(x)=x$.

Fibonacci-Like sequence [10] is defined by

$S_{n}=S_{n-1}+S_{n-2}, n \geq 2$ with $S_{0}=2$ and $S_{1}=2$.

There is a long tradition of using matrices to study Fibonacci, Lucas, Pell, Pell Lucas, Modified Pell, Jacobsthal, Jacobsthal Lucas numbers and polynomials. Many authors [1, 3, 5, 6, 7, 8, 9] were studied properties of these sequences and polynomials by matrix methods.

In this paper, Fibonacci-Like polynomials (FLP) is introducing with some basic identities and derived by standard method.

\section{Preliminaries of Fibonacci-Like Polynomials}

In this section, Fibonacci-Like polynomials is present with some basic results.

Fibonacci-Like polynomials (FLP) is introduced and defined by the recurrence relation: 
$s_{n}(x)=x s_{n-1}(x)+s_{n-2}(x), n \geq 2$ with initial terms $s_{0}(x)=2$ and $s_{1}(x)=2 x$.

A few Fibonacci-Like polynomials are as follows:

$s_{1}(x)=2 x$,

$s_{2}(x)=2 x^{2}+2$,

$s_{3}(x)=2 x^{3}+4 x$,

$s_{4}(x)=2 x^{4}+6 x^{2}+2$,

$s_{5}(x)=2 x^{5}+8 x^{3}+6 x, \ldots$

For $x=1$, we obtain Fibonacci-Like sequence (1.3).

The characteristic equation of recurrence relation $(2.1)$ is $\lambda^{2}-x \lambda-1=0$.

It gives two real roots $\alpha=\frac{x+\sqrt{x^{2}+4}}{2}$ and $\beta=\frac{x+\sqrt{x^{2}+4}}{2}$.

Also, $\alpha \beta=-1, \alpha+\beta=x, \alpha-\beta=\sqrt{x^{2}+4}$.

Generating function of Fibonacci-Like polynomials is

$\sum_{n=0}^{\infty} s_{n}(x) t^{n}=\frac{2}{\left(1-x t-t^{2}\right)}=2\left(1-x t-t^{2}\right)^{-1}$.

Its Hypergeometric form is

$\sum_{n=0}^{\infty} s_{n}(x) \frac{t^{n}}{n !}=2 e_{2}^{x t} F_{1}\left(n+1,1 ; 1 ; t^{2}\right)$.

Binet's formula of Fibonacci-Like Polynomials is defined by

$s_{n}(x)=A \alpha^{n}+B \beta^{n}$, where $A=\frac{2(x-\beta)}{\alpha-\beta}$ and $B=\frac{2(\alpha-x)}{\alpha-\beta}$

Matrix Representation

Define $Q_{s}$, a $2 \times 2$ order matrix by

$Q_{S}=\left[\begin{array}{lc}2\left(x^{2}+1\right) & 2 x \\ 2 x & 2\end{array}\right]=\left[\begin{array}{ll}s_{2}(x) & s_{1}(x) \\ s_{1}(x) & s_{0}(x)\end{array}\right]$

By induction,

$Q_{s}^{n}=2^{n-1}\left[\begin{array}{ll}s_{2 n}(x) & s_{2 n-1}(x) \\ s_{2 n-1}(x) & s_{2 n-2}(x)\end{array}\right]$ for $n \geq 1$, where $s_{2 n}(x)$ is Fibonacci-Like polynomials.

\section{Some Identities of Fibonacci-Like Polynomials}

In this section, some identities like Catalan's, Cassini's, d'Ocagne's identities etc. are present with solution by Binet's formula or explicit sum formula or generating function or respective matrix.

Theorem (3.1): Let $Q_{s}$ be a $2 \times 2$ matrix, then for all integers $n \geq 1$,

$Q_{s}^{n}=2^{n}\left[\begin{array}{ll}f_{2 n+1}(x) & f_{2 n}(x) \\ f_{2 n}(x) & f_{2 n-1}(x)\end{array}\right]$.

By induction, it can easily be proved. 
Theorem (3.2): Let $Q_{s}$ be a $2 \times 2$ matrix, then for all integers $m \geq 1, n \geq 1$,

(i) $2 s_{2(m+n)}(x)=s_{2 m-1}(x) s_{2 n-1}(x)+s_{2 m}(x) s_{2 n}(x)$

(ii) $2 s_{2(m+n)-1}(x)=s_{2 m-1}(x) s_{2 n-2}(x)+s_{2 m}(x) s_{2 n-1}(x)$

(iii) $2 s_{2(m+n)-1}(x)=s_{2 m-2}(x) s_{2 n-1}(x)+s_{2 m-1}(x) s_{2 n}(x)$

(iv) $2 s_{2(m+n)-2}(x)=s_{2 m-2}(x) s_{2 n-2}(x)+s_{2 m-1}(x) s_{2 n-1}(x)$.

Proof: By (2.8), it follows that

$Q_{s}^{m+n}=2^{m+n-1}\left[\begin{array}{ll}s_{2(m+n)}(x) & s_{2(m+n)-1}(x) \\ s_{2(m+n)-1}(x) & s_{2(m+n)-2}(x)\end{array}\right]$.

Since $Q_{s}^{m+n}=Q_{s}^{m} Q_{s}^{n}$, therefore we have

$Q_{s}^{m+n}=2^{m+n-2}\left[\begin{array}{ll}s_{2 m}(x) s_{2 n}(x)+s_{2 m-1}(x) s_{2 n-1}(x) & s_{2 m}(x) s_{2 n-1}(x)+s_{2 m-1}(x) s_{2 n-2}(x) \\ s_{2 m-1}(x) s_{2 n}(x)+s_{2 m-2}(x) s_{2 n-1}(x) & s_{2 m-1}(x) s_{2 n-1}(x)+s_{2 m-2}(x) s_{2 n-2}(x)\end{array}\right]$.

Equating corresponding elements of (3.2) and (3.3), we obtain required results.

Theorem (3.3): Let $Q_{s}$ be a $2 \times 2$ matrix, then for integers $m \geq 1, n \geq 1$,

(i) $s_{2(m+n)}(x)=2\left\{f_{2 m}(x) f_{2 n}(x)+f_{2 m+1}(x) f_{2 n+1}(x)\right\}$

(ii) $s_{2(m+n)-1}(x)=2\left\{f_{2 m}(x) f_{2 n-1}(x)+f_{2 m+1}(x) f_{2 n}(x)\right\}$

(iii) $s_{2(m+n)}(x)=2\left\{f_{2 m-1}(x) f_{2 n}(x)+f_{2 m}(x) f_{2 n+1}(x)\right\}$

(iv) $s_{2(m+n)-1}(x)=2\left\{f_{2 m-1}(x) f_{2 n-1}(x)+f_{2 m}(x) f_{2 n}(x)\right\}$.

Theorem (3.4): Let $Q_{s}$ be a $2 \times 2$ matrix, then for all integers $m \geq 1, n \geq 1$,

(i) $f_{2(m+n)+1}(x)=f_{2 m}(x) f_{2 n}(x)+f_{2 m+1}(x) f_{2 n+1}(x)$

(ii) $f_{2(m+n)}(x)=f_{2 m}(x) f_{2 n-1}(x)+f_{2 m+1}(x) f_{2 n}(x)$

(iii) $f_{2(m+n)}(x)=f_{2 m-1}(x) f_{2 n}(x)+f_{2 m}(x) f_{2 n+1}(x)$

(iv) $f_{2(m+n)-1}(x)=f_{2 m-1}(x) f_{2 n-1}(x)+f_{2 m}(x) f_{2 n}(x)$.

Theorem (3.5): Let $Q_{s}$ be a $2 \times 2$ matrix, then for all integers $m \geq 1, n \geq 1$,

(i) $4 f_{2(m+n)+1}(x)=s_{2 m-1}(x) s_{2 n-1}(x)+s_{2 m}(x) s_{2 n}(x)$

(ii) $4 f_{2(m+n)}(x)=s_{2 m-1}(x) s_{2 n-2}(x)+s_{2 m}(x) s_{2 n-1}(x)$

(iii) $4 f_{2(m+n)}(x)=s_{2 m-2}(x) s_{2 n-1}(x)+s_{2 m-1}(x) s_{2 n}(x)$

(iv) $4 f_{2(m+n)-1}(x)=s_{2 m-2}(x) s_{2 n-2}(x)+s_{2 m-1}(x) s_{2 n-1}(x)$.

The proof of above identities can be given easily by (2.8) and (3.1) similar as theorem (3.2).

Theorem (3.6): The summation of Fibonacci-Like polynomials is given by

$\sum_{k=0}^{n-1} s_{k}(x)=\frac{s_{n}(x)+s_{n-1}(x)-2}{x}$.

Proof. By Binet's formula (2.6), obtain

$$
\begin{aligned}
\sum_{k=0}^{n-1} s_{k}(x) & =\sum_{k=0}^{n-1}\left[A \alpha^{k}+B \beta^{k}\right]=A \sum_{k=0}^{n-1} \alpha^{k}+\sum_{k=0}^{n-1} \beta^{k}=A\left[\frac{1-\alpha^{n}}{1-\alpha}\right]+B\left[\frac{1-\beta^{n}}{1-\beta}\right] \\
& =\frac{(A+B)-(A \beta+B \alpha)-\left(A \alpha^{n}+B \beta^{n}\right)+\alpha \beta\left(A \alpha^{n-1}+B \beta^{n-1}\right)}{1-(\alpha+\beta)+\alpha \beta} \\
\sum_{k=0}^{n-1} s_{k}(x) & =\frac{2-0-s_{n}(x)+(-1) s_{n-1}(x)}{1-x+(-1)}=\frac{s_{n}(x)+s_{n-1}(x)-2}{x}
\end{aligned}
$$


Theorem (3.7): The explicit sum formula for Fibonacci-Like polynomials is given by

$$
s_{n}(x)=2 \sum_{k=0}^{\left[\frac{n}{2}\right]}\left(\begin{array}{c}
n-k \\
k
\end{array}\right) x^{n-2 k} \text {. }
$$

Proof: By generating function (2.4), obtain

$$
\begin{aligned}
& \sum_{n=0}^{\infty} s_{n}(x) t^{n}=2\left(1-x t-t^{2}\right)^{-1}=2[1-(x+t) t]^{-1} \\
& =2 \sum_{n=0}^{\infty}(x+t)^{n} t^{n}=2 \sum_{n=0}^{\infty} t^{n} \sum_{k=0}^{n}\left(\begin{array}{l}
n \\
k
\end{array}\right) x^{n-k} t^{k} \\
& =2 \sum_{n=0}^{\infty} \sum_{k=0}^{n} \frac{n !}{k ! n-k !} x^{n-k} t^{k+n}=2 \sum_{n=0}^{\infty} \sum_{k=0}^{\infty} \frac{n+k !}{k ! n !} x^{n} t^{n+2 k} \\
& =2 \sum_{n=0}^{\infty} \sum_{k=0}^{\left[\frac{n}{2}\right]} \frac{n-k !}{k ! n-2 k !} x^{n-2 k} t^{n}
\end{aligned}
$$

Equating the coefficient of ' $t^{n}$, on both sides, obtain the required explicit formula.

Theorem (3.8) (Catalan's Identity): Let $s_{n}(x)$ be the $n^{\text {th }}$ Fibonacci-Like polynomial, then $s_{n}^{2}(x)-s_{n+r}(x) s_{n-r}(x)=(-1)^{n-r+1} s_{r-1}^{2}(x)$ for $n>r \geq 1$.

Proof: Using Binet's formula (2.6), obtain

$$
\begin{aligned}
& S_{n}^{2}(x)-s_{n+r}(x) s_{n-r}(x) \\
& =\left(A \alpha^{n}+B \beta^{n}\right)^{2}-\left(A \alpha^{n+r} B \beta^{n+r}\right)\left(A \alpha^{n-r}+B \beta^{n-r}\right) \\
& =A B(\alpha \beta)^{n}\left(2-\alpha^{r} \beta^{-r}-\alpha^{-r} \beta^{r}\right) \\
& =A B(-1)^{n} \frac{(-1)}{(-1)^{r}}\left(\alpha^{r}-\beta^{r}\right)^{2} \\
& =\frac{4}{(\alpha-\beta)^{2}}(-1)^{n-r+1}\left(\alpha^{r}-\beta^{r}\right)^{2} \\
& =4(-1)^{n-r+1}\left(\frac{\alpha^{r}-\beta^{r}}{\alpha-\beta}\right)^{2}
\end{aligned}
$$

Since $\frac{\alpha^{r}-\beta^{r}}{\alpha-\beta}=\frac{2 x s_{r}(x)-2 s_{r+1}(x)}{-4}=\frac{s_{r-1}(x)}{2}$, obtain

$$
s_{n}^{2}(x)-s_{n+r}(x) s_{n-r}(x)=(-1)^{n-r+1} s_{r-1}^{2}(x) .
$$

Corollary (3.9) (Cassini's Identity): Let $s_{n}(x)$ be the $n^{\text {th }}$ Fibonacci-Like polynomial, then $s_{n}^{2}(x)-s_{n+1}(x) s_{n-1}(x)=4(-1)^{n}, n \geq 1$.

Proof: If $r=1$ in the Catalan's identity, then obviously proved.

If $x=1$, then obtain Cassini's identity for Fibonacci-Like sequences.

Theorem (3.10) (d'Ocagne's Identity): Let $s_{n}(x)$ be the $n^{\text {th }}$ Fibonacci-Like polynomial, then $s_{m}(x) s_{n+1}(x)-s_{m+1}(x) s_{n}(x)=2(-1)^{n+1} s_{m-n-1}(x), m>n \geq 0$.

Proof: Using Binet's formula (2.6), obtain

$$
\begin{aligned}
s_{m}(x) s_{n+1}(x)-s_{m+1}(x) s_{n}(x) & =\left(A \alpha^{m}+B \beta^{m}\right)\left(A \alpha^{n+1}+B \beta^{n+1}\right)-\left(A \alpha^{m+1}+B \beta^{m+1}\right)\left(A \alpha^{n}+B \beta^{n}\right) \\
= & A B\left(\alpha^{m} \beta^{n+1}+\alpha^{n+1} \beta^{m}-\alpha^{n} \beta^{m+1}-\alpha^{m+1} \beta^{n}\right) \\
= & A B(\alpha \beta)^{n}\left[\beta\left(\alpha^{m-n}-\beta^{m-n}\right)-\alpha\left(\alpha^{m-n}-\beta^{m-n}\right)\right] \\
= & A B(-1)^{n}(\beta-\alpha)\left(\alpha^{m-n}-\beta^{m-n}\right) \\
= & \frac{4}{(\alpha-\beta)^{2}}(-1)^{n+1}(\alpha-\beta)\left(\alpha^{m-n}-\beta^{m-n}\right)
\end{aligned}
$$




$$
\begin{aligned}
& \qquad=4(-1)^{n+1}\left(\frac{\alpha^{m-n}-\beta^{m-n}}{\alpha-\beta}\right) \\
& \text { From } \frac{\alpha^{m-n}-\beta^{m-n}}{\alpha-\beta}=\frac{2 x s_{m-n}(x)-2 s_{m-n+1}(x)}{2 x s_{1}(x)-2 s_{2}(x)}=\frac{s_{m-n-1}(x)}{2}, \text { obtain } \\
& s_{m}(x) s_{n+1}(x)-s_{m+1}(x) s_{n}(x)=2(-1)^{n+1} s_{m-n-1}(x) .
\end{aligned}
$$

Hence, $s_{m}(x) s_{n+1}(x)-s_{m+1}(x) s_{n}(x)=2(-1)^{n+1} s_{m-n-1}(x), m \geq 1, n \geq 0, m>n$.

Theorem (3.11) (Generalized Identity): Let $s_{n}(x)$ be the $n^{\text {th }}$ Fibonacci-Like polynomial, then $s_{m}(x) s_{n}(x)-s_{m-r}(x) s_{n+r}(x)=(-1)^{m-r+1} s_{r-1}(x) s_{n-m+r-1}(x), n>m \geq r \geq 1$.

Proof. The proof can be given by Binet's formula.

The identity (3.9) is generalization of Catalan's, Cassini's and d'Ocagne's identities.

- If $m=m=n+1$ in identity (3.9), then obtain Catalan's identity (3.6).

- If $m=n+1, n=n-1$ and $r=1$ in identity (3.9), then obtain Cassini's identity (3.7).

- If , $n=m, \quad m=n+1$ and $r=1$ in identity (3.9), then obtain d'Ocagne's identity (3.8).

- For $x=1$, we obtain Catalan's, Cassini's and d'Ocagne's identities of Fibonacci-Like numbers.

Theorem (3.12): For positive integer $n \geq 0$, prove that

$$
s_{n}(x)=2 x_{2}^{n} F_{1}\left(\frac{-n}{2}, \frac{-n+1}{2} ;-n ; \frac{-4}{x^{2}}\right) \text {. }
$$

Proof: By explicit sum formula (3.5), it follows that

$$
\begin{aligned}
s_{n}(x)= & 2 x^{n} \sum_{k=0}^{[n / 2]} \frac{n-k !}{k ! n-2 k !} x^{-2 k} \\
& =2 x^{n} \sum_{k=0}^{[n / 2]} \frac{(-1)^{k}(1)_{n}(-n)_{2 k}}{(-n)_{k}(-1)^{2 k}(1)_{n}} \frac{x^{-2 k}}{k !} \\
& =2 x^{n} \sum_{k=0}^{[n / 2]} \frac{(-1)^{k} 2^{2 k}\left(\frac{-n}{2}\right)_{k}\left(\frac{-n+1}{2}\right)_{k}}{(-n)_{k}(-1)^{2 k}} \frac{x^{-2 k}}{k !} \\
& =2 x^{n} \sum_{k=0}^{[n / 2]} \frac{\left(\frac{-n}{2}\right)_{k}\left(\frac{-n+1}{2}\right)_{k} \frac{\left(\frac{-4}{x^{2}}\right)^{k}}{k !}}{(-n)_{k}}
\end{aligned}
$$

Hence, $s_{n}(x)=2 x_{2}^{n} F_{1}\left(\frac{-n}{2}, \frac{-n+1}{2} ;-n ; \frac{-4}{x^{2}}\right)$.

Theorem (3.13): For positive integer $n \geq 0$, prove that $\sum_{n=0}^{\infty}(c)_{n} s_{n}(x) \frac{t^{n}}{n !}=2(1-x t)^{-c}{ }_{3} F_{2}\left(\frac{c}{2}, \frac{c+1}{2}, n+1 ; \frac{n+1}{2} ; \frac{n+2}{2} ; \frac{t^{2}}{(1-x t)^{2}}\right)$.

Proof: Multiplying both sides of the explicit sum formula (3.5) by $(c)_{n} \frac{t^{n}}{n !}$ and summing between the limit $n=0$ to $n=\infty$, obtain 


$$
\begin{aligned}
& \sum_{n=0}^{\infty}(c)_{n} s_{n}(x) \frac{t^{n}}{n !}=2 \sum_{n=0}^{\infty} \sum_{k=0}^{\left[\frac{n}{2}\right]} \frac{n-k !}{k ! n-2 k !}(c)_{n} x^{n-2 k} \frac{t^{n}}{n !} \\
& =2 \sum_{n=0}^{\infty} \sum_{k=0}^{\infty} \frac{n+k !}{k ! n ! n+2 k !}(c)_{n+2 k} x^{n} t^{n+2 k} \\
& =2\left\{\sum_{n=0}^{\infty}(c+2 k)_{n} \frac{(x t)^{n}}{n !}\right\} \sum_{k=0}^{\infty} \frac{n+k !}{k ! n+2 k !}(c)_{2 k} t^{2 k} \\
& =2 \sum_{k=0}^{\infty}(1-x t)^{-(c+2 k)} \frac{n+k !}{k ! n+2 k !}(c)_{2 k} t^{2 k} \text {, } \\
& \sum_{n=0}^{\infty}(c)_{n} s_{n}(x) \frac{t^{n}}{n !}=2(1-x t)^{-c} \sum_{k=0}^{\infty} \frac{n+k !}{k ! n+2 k !}(c)_{2 k}\left[\frac{t^{2}}{(1-x t)^{2}}\right]^{k} \\
& =2(1-x t)^{-c} \sum_{k=0}^{\infty} \frac{n+k !}{n+2 k !} 2^{2 k}\left(\frac{c}{2}\right)_{k}\left(\frac{c+1}{2}\right)_{k}\left[\frac{t^{2}}{(1-x t)^{2}}\right]^{k} / k ! \\
& =2(1-x t)^{-c} \sum_{k=0}^{\infty} \frac{(n+1)_{k}}{(n+1)_{2 k}} 2^{2 k}\left(\frac{c}{2}\right)_{k}\left(\frac{c+1}{2}\right)_{k}\left[\frac{t^{2}}{(1-x t)^{2}}\right]^{k} / k ! \text {, } \\
& =2(1-x t)^{-c} \sum_{k=0}^{\infty} \frac{\left(\frac{c}{2}\right)_{k}\left(\frac{c+1}{2}\right)_{k}(n+1)_{k}}{\left(\frac{n+1}{2}\right)_{k}\left(\frac{n+2}{2}\right)_{k}}\left[\frac{t^{2}}{(1-x t)^{2}}\right]^{k} / k !
\end{aligned}
$$

\section{Conclusion}

In this paper, Fibonacci-Like polynomials (FLP) is introduced and presented some basic results. Further some basic identities (like Catalan's, Cassini's, d'Ocagne's etc.) are describe with derivation by standard method. The concept of Fibonacci-Like polynomials can be extended in two and three variables with basic results and identities.

\section{Acknowledgement}

We would like to thank the anonymous referee for numerous helpful suggestions.

\section{References}

[1] Demiriturk, B., Fibonacci and Lucas Sums by Matrix Methods, Int. Math. Forum, Vol. 5, No. 3 (2010), 99-107.

[2] Garth, D., Mills, D., Mitchell, P., Polynomials Generated by the Fibonacci Sequence, Journal of Integer Sequences, Vol. 10 (2007), Article 07.6.8.

[3] Horadam, A. F., Mahon, J. M., Pell and Pell-Lucas Polynomials, Fibonacci Quart., Vol. 23, No. 1 (1985), 7-20.

[4] Horzum, T., Kocer, E. G., On Some Properties of Horadam Polynomials, Int. Math. Forum, Vol. 4, No. 25 (2009), $1243-1252$.

[5] Koken, F., Bozkurt, D., On the Jacobsthal Numbers by Matrix Methods, Int. J. Contemp. Math. Sciences, Vol. 3, No. 13 (2008), 605-614

[6] Koken, F., Bozkurt, D., On the Jacobsthal Numbers by Matrix Methods, Int. J. Contemp. Math. Sciences, Vol. 3 , No. 33 (2008), $1629-1633$.

[7] Koshy, T., Fibonacci and Lucas numbers with Applications, John Wiley and Sons. New York, 2001.

[8] Lupas, A., A Guide of Fibonacci and Lucas Polynomial, Octagon Mathematics Magazine, Vol. 7, No.1 (1999), $2-12$.

[9] Silvester, J. R., Fibonacci Properties by Matrix Methods, Mathematical Gazette, 63 (1979), $188-191$.

[10] Singh, B., Sikhwal, O., Bhatnagar, S., Fibonacci-Like Sequence and its Properties, Int. J. Contemp. Math. Sciences, Vol. 5, No. 18 (2010), $859-868$. 